\title{
Role of local structural distortion in driving ferroelectricity in $\mathrm{GdCrO}_{3}$
}

\author{
Sudipta Mahana, ${ }^{1,2}$ U. Manju, ${ }^{3}$ Pronoy Nandi, ${ }^{1,2}$ Edmund Welter, ${ }^{4}$ K. R. Priolkar, ${ }^{5}$ and D. Topwal ${ }^{1,2, *}$ \\ ${ }^{1}$ Institute of Physics, Sachivalaya Marg, Bhubaneswar 751005, India \\ ${ }^{2}$ Homi Bhabha National Institute, Training School Complex, Anushakti Nagar, Mumbai 400085, India \\ ${ }^{3}$ CSIR-Institute of Minerals and Materials Technology, Bhubaneswar 751013, India \\ ${ }^{4}$ Deutsches Elektronen-Synchrotron, Hamburg 22607, Germany \\ ${ }^{5}$ Department of Physics, Goa University, Taleigao Plateau, Goa 403206, India
}

(Received 27 March 2018; revised manuscript received 25 May 2018; published 26 June 2018)

\begin{abstract}
Temperature-dependent synchrotron x-ray diffraction and extended x-ray absorption fine structure (EXAFS) studies were performed to understand the role of structural characteristics in driving the magnetoelectric multiferroic properties of $\mathrm{GdCrO}_{3}$. The results suggest that the distortion in the structure appears to be associated with the off-center displacement of Gd atoms together with octahedral rotations via displacement of the oxygen ions in $\mathrm{GdCrO}_{3}$. Further, a comparative EXAFS study of $\mathrm{GdCrO}_{3}$ with a similar system, $\mathrm{YCrO}_{3}$, suggests that the oxygen environment of $\mathrm{Gd}$ in $\mathrm{GdCrO}_{3}$ is different from that of $\mathrm{Y}$ in $\mathrm{YCrO}_{3}$, which results in an orthorhombic $P n a 2_{1}$ structure in $\mathrm{GdCrO}_{3}$ in contrast to the monoclinic $P 2_{1}$ structure in $\mathrm{YCrO}_{3}$.
\end{abstract}

DOI: 10.1103/PhysRevB.97.224107

\section{INTRODUCTION}

Magnetoelectric multiferroics have drawn great interest in recent years due to their multifunctionality for a wide variety of potential device applications in modern technologies [1-4]. The family of rare-earth chromites $\left(\mathrm{RCrO}_{3}\right)$ has been recognized as promising systems for multiferroicity at reasonably high temperatures [5-8]. But the conflicting observations of the ferroelectric behavior at relatively high temperature and the average centrosymmetric lattice (Pbnm) and magnetic structure ( $G$-type) in these systems remained a puzzling issue in this series of compounds [5,9]. $\mathrm{GdCrO}_{3}$, a member of the $R \mathrm{CrO}_{3}$ family, shows magnetic and ferroelectric transitions simultaneously at around $169 \mathrm{~K}\left(T_{M}\right)$ [5] in contrast to $\mathrm{YCrO}_{3}$ having a ferroelectric transition at $473 \mathrm{~K}$ and a magnetic transition at $140 \mathrm{~K}$ [10]. In addition, the strength of the electric polarization in $\mathrm{GdCrO}_{3}$ is one order of magnitude less than that of $\mathrm{YCrO}_{3}[5,10]$. Our recent report on temperature-dependent $\mathrm{x}$-ray diffraction (XRD) studies along with first-principles density-functional-theory calculations showed that $\mathrm{GdCrO}_{3}$ possesses noncentrosymmetric orthorhombic $P n a 2_{1}$ structure [11], in contrast to the monoclinic $P 2_{1}$ (noncentrosymmetric) structure in $\mathrm{YCrO}_{3}$ as reported earlier [10,12], which leads to a weaker polar property in $\mathrm{GdCrO}_{3}$ compared to $\mathrm{YCrO}_{3}$. Phonon instability studies in the high-symmetry cubic perovskite structure showed that there is a strong similarity between $\mathrm{GdCrO}_{3}$ and $\mathrm{YCrO}_{3}$ as the polar distortion in both the systems is associated with the $R$-ion displacement $[10,11,13]$. Furthermore, as the ferroelectric instability is very weak in these systems, the local noncentrosymmetry has been suggested to play a crucial role in driving ferroelectricity $[10,12]$. Thus, it is essential to study the short-range structural order in these systems to understand the origin of their ferroelectric properties. Since

*dinesh.topwal@iopb.res.in, dinesh.topwal@gmail.com extended $\mathrm{x}$-ray absorption fine structure (EXAFS) is a powerful tool for local structure investigations, we performed detailed EXAFS studies in conjunction with temperature-dependent XRD to understand the structure of $\mathrm{GdCrO}_{3}$. These studies show that a strong correlation exists between the presence of local distortion and its implication in the ferroelectric ordering and the global structure of the system. Further, we also discuss a comparative EXAFS study of $\mathrm{GdCrO}_{3}$ with the similar chromite system, $\mathrm{YCrO}_{3}$.

\section{EXPERIMENTAL DETAILS}

Polycrystalline samples of $\mathrm{GdCrO}_{3}\left(\mathrm{YCrO}_{3}\right)$ were prepared by the solid-state synthesis technique using stoichiometric proportions of $\mathrm{Gd}_{2} \mathrm{O}_{3}\left(\mathrm{Y}_{2} \mathrm{O}_{3}\right)$ and $\mathrm{Cr}_{2} \mathrm{O}_{3}$ and details are described elsewhere [14,15]. Phase purity of the samples was confirmed by powder XRD measurements carried out in a D8 advanced diffractometer equipped with $\mathrm{Cu} K_{\alpha}$ radiation. Temperaturedependent XRD measurements were carried out at the XRD1 beamline at the Elettra synchrotron radiation facility using photons with a wavelength of $0.85507 \AA$. Rietveld refinements of the diffraction patterns were performed using the FULLPROF package. Temperature-dependent EXAFS measurements were carried out at P-65 beamline at PETRA-III synchrotron source, DESY, Hamburg, Germany. Both incident $\left(I_{0}\right)$ and transmitted $\left(I_{t}\right)$ photon intensities were recorded simultaneously using ionization chambers filled with appropriate gases at $\mathrm{Gd} L_{3}$ $(7243 \mathrm{eV})$ and $\mathrm{Cr} K(5989 \mathrm{eV})$ edges in $\mathrm{GdCrO}_{3}$ and $\mathrm{Y} K$ $(17038 \mathrm{eV})$ and $\mathrm{Cr} K$ edges in $\mathrm{YCrO}_{3}$. The raw data collected were background subtracted and normalized to extract EXAFS signals through a series of steps using the ATHENA software [16]. Thereafter, the fitting of the EXAFS spectrum with a specific model obtained from basic crystallographic information was carried out using the ARTEMIS software [16]. The software computes the theoretical spectrum from the given model using the ATOMS and FEFF6 programs [16-18]. 


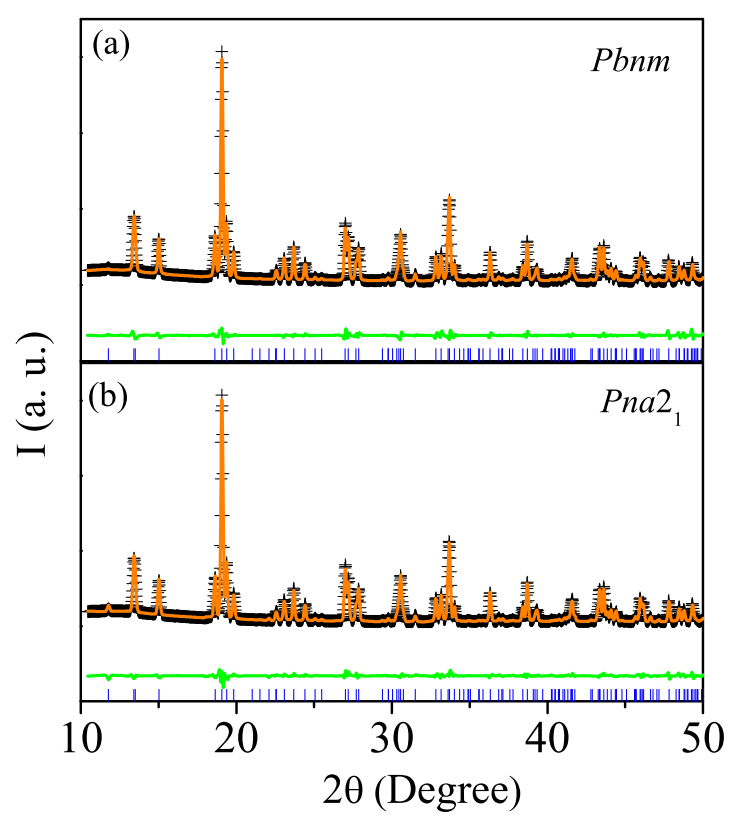

FIG. 1. Room-temperature x-ray powder diffraction patterns (symbols) of $\mathrm{GdCrO}_{3}$ with corresponding refinement patterns (solid curve) using the (a) Pbnm and (b) Pna2 $2_{1}$ space groups.

\section{RESULTS AND DISCUSSION}

Rietveld refinement of the XRD patterns of $\mathrm{GdCrO}_{3}$ were performed using both centrosymmetric Pbnm and noncentrosymmetric $P n a 2_{1}$ space groups throughout the entire (studied) temperature range $(100-300 \mathrm{~K})$. It was found that both the space groups fit with better goodness-of-fit parameters in the entire range. The XRD pattern acquired at $300 \mathrm{~K}$ along with corresponding Rietveld refinement data using $\mathrm{Pbnm}$ and $P n a 2_{1}$ space groups are depicted in Figs. 1(a) and 1(b), respectively. The reliability parameters obtained for the $P b n m$ space group are $R_{w} \sim 0.084, R_{\text {expt }} \sim 0.041$, and $\chi^{2} \sim 4.16$ and the corresponding parameters for the $P n a 2_{1}$ structure are $R_{w} \sim 0.083, R_{\text {expt }} \sim 0.041$, and $\chi^{2} \sim 4.37$. The similarity in reliability parameters is possibly due to the small structural changes between the two space groups as $P n a 2_{1}$ is a subgroup of Pbnm. To verify the structure, we used the program PLATON $[19,20]$ and found that the symmetry of the structure is better described in $P n a 2_{1}$ throughout the entire temperature range. The noncentrosymmetric $P n a 2_{1}$ structure in $\mathrm{GdCrO}_{3}$ was also supported by density-functional theory calculations, favoring the polar nature of the system as reported earlier [11].

In addition to $\mathrm{x}$-ray diffraction studies, temperaturedependent EXAFS measurements were also performed to extract information about the local structure around selected atoms. The EXAFS technique is useful to provide valuable information about the structural peculiarities and allows one to verify different structural models. Figures 2(a) and 2(b) show $k^{3}$-weighted EXAFS data in $k$ space at $\mathrm{Cr} K$ and $\mathrm{Gd} L_{3}$ edges, respectively, acquired at room temperature for $\mathrm{GdCrO}_{3}$, along with corresponding fittings superimposed on it. The magnitudes of Fourier transforms of the corresponding spectra along with fittings are shown in Figs. 2(c) and 2(d), respectively. Various contributions in different regions of the spectra are marked in the figures. The scattering contributions
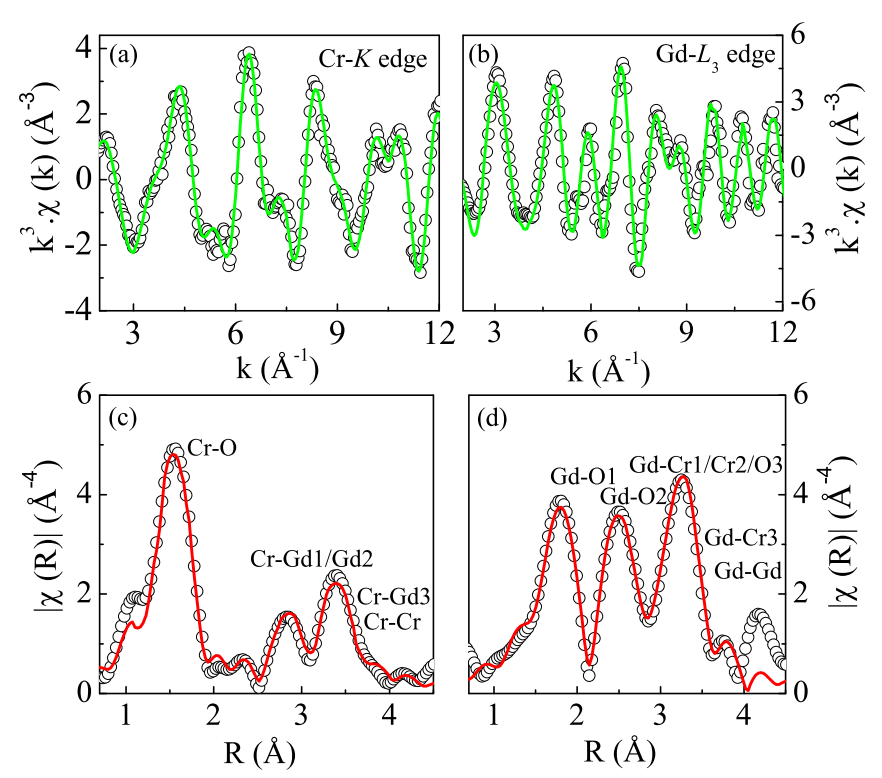

FIG. 2. $k^{3}$-weighted EXAFS data in $k$ space (open circles) at (a) the $\mathrm{Cr} K$ edge and (b) the $\mathrm{Gd} L_{3}$ edge acquired at room temperature for $\mathrm{GdCrO}_{3}$, along with corresponding fittings (solid lines). The magnitudes of Fourier transforms spectra (open circles) of $\mathrm{Cr} K$ edge and $\mathrm{Gd} L_{3}$ edge along with the corresponding fitting (solid lines) are shown in (c) and (d) respectively. Various contributions in different regions are marked in the figures.

for atomic shells were derived considering both Pbnm and $P n a 2_{1}$ crystal structures and in both cases the EXAFS data fit well throughout the entire (measured) temperature range having an $R$ factor of $\sim 0.01$ for the $\mathrm{Cr} K$ edge and $\sim 0.009$ for the $\mathrm{Gd} L_{3}$ edges. This corroborates that distortions in the structure are very small, in agreement with the XRD results as discussed above. For the $\mathrm{Cr} K$ edge, fits were confined to a $k$ range of $3<k<12 \AA^{-1}$ and an $R$ range of $1.2<R<4 \AA$. In this region $\mathrm{Cr} K$ EXAFS originates from scattering of photoelectrons from the nearest-neighbor octahedral oxygens of $\mathrm{Cr}$, three $\mathrm{Gd}$ subshells with two, four, and two coordination numbers, respectively, and a $\mathrm{Cr}-\mathrm{Cr}$ coordination shell with four atoms and some multiple scattering contributions. The fitting of the Gd $L_{3}$ edge was done in the $k$ range of $3<$ $k<12.5 \AA^{-1}$ and an $R$ range of $1.1<R<4 \AA$, to model $\mathrm{Gd}-\mathrm{O}$ and $\mathrm{Gd}-\mathrm{Cr}$ distributions. The Gd-O distribution is more complex, consisting of three subshells with four, two, and six oxygens, respectively. The Gd-Cr contribution is also split into three subshells with two, four, and two $\mathrm{Cr}$ atoms. Additionally, a single Gd-Gd shell is considered with coordination number four. During the fitting procedure, the coordination number was kept fixed, while bond length and mean-square relative displacement (MSRD) $\left[\sigma^{2}=\left\langle(r-\langle r\rangle)^{2}\right\rangle\right]$ were used as free parameters. It is seen that MSRD for short (O1) and long (O3) Gd-O bonds are highly correlated; therefore, there is a single $\sigma^{2}$ for them and a separate one for intermediate oxygens $(\mathrm{O} 2)$. Similarly, two $\sigma^{2}$ were chosen for Gd-Cr bonds: one for short and long bonds in the $b c$ plane and another for intermediate bonds. $\sigma^{2}$ are the most sensitive to modes contributing to radial motions, basically depending only on the local vibrational structure [17]. 


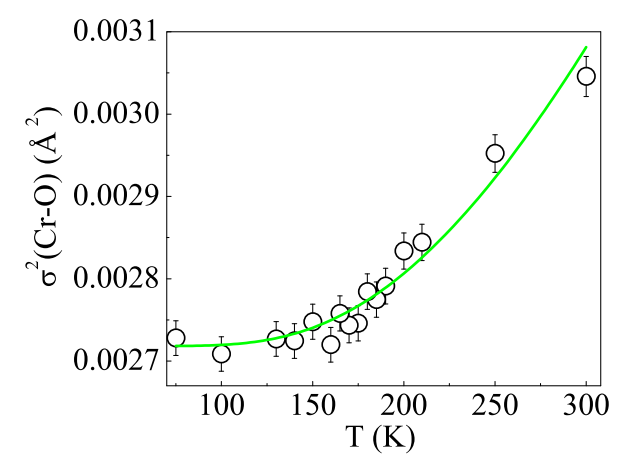

FIG. 3. Temperature dependence of mean-square relative displacement (MSRD) $\left(\sigma^{2}\right)$ of $\mathrm{Cr}-\mathrm{O}$ bond correlation of $\mathrm{GdCrO}_{3}$. The solid line represents the fitting using the Einstein model [Eq. (1)].

The temperature dependencies of $\sigma^{2}$ for all the shells were fitted with the Einstein model, which considers the bond vibrations as harmonic oscillations with a single effective frequency proportional to the Einstein temperature, $\theta_{E}$, as given by the relation $[17,21]$

$$
\sigma^{2}(T)=\sigma_{0}^{2}+\left(\frac{\hbar^{2}}{2 \mu k_{B} \theta_{E}}\right) \operatorname{coth}\left(\frac{\theta_{E}}{2 T}\right),
$$

where $\sigma_{0}^{2}$ is the static contribution, $T$ is in kelvins, and $\mu$ is the reduced mass of the bond pair. The Einstein temperature $\theta_{E}$ is a measure of the stiffness of the bonds. Fitting of the temperature dependence of $\sigma^{2}$ using the Einstein model for $\mathrm{Cr}-\mathrm{O}$ bond correlation is shown in Fig. 3. The best fitting yields $\sigma_{0}^{2}=0.0012(4)$ and $\theta_{E}=818(11) \mathrm{K}$. The large value

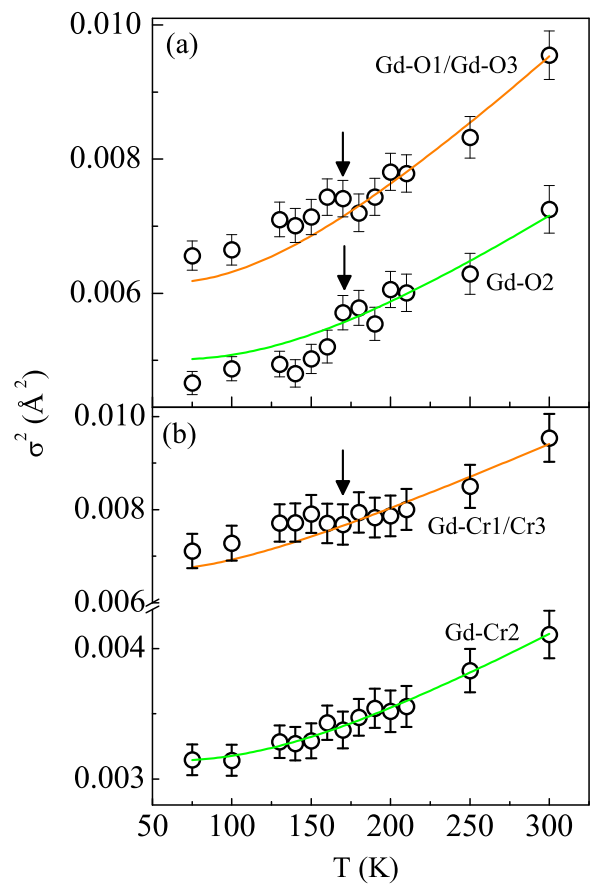

FIG. 4. (a) Temperature dependence of MSRD $\left(\sigma^{2}\right)$ of (a) Gd-O bond correlations and (b) Gd-Cr bond correlations in $\mathrm{GdCrO}_{3}$. The solid lines represent the fitting using the Einstein model [Eq. (1)]. The arrows indicate the magnetic/ferroelectric transition temperature. of $\theta_{E}$ indicates the rigidness of the $\mathrm{CrO}_{6}$ octahedra [22]. Furthermore, no anomalous change in the $\sigma^{2}$ is observed below $169 \mathrm{~K}$, where both magnetic and ferroelectric ordering are observed.

The fitting of the temperature dependence of $\sigma^{2}$ for Gd-O subshells using Eq. (1) is shown in Fig. 4(a). Fitting yielded $\sigma_{0}^{2}=0.0015(4)$ and $\theta_{E}=391(15) \mathrm{K}$ for Gd-O1/O3 bond correlations and $\sigma_{0}^{2}=0.0011(4)$ and $\theta_{E}=458(26) \mathrm{K}$ for Gd$\mathrm{O} 2$ bond correlation. The relatively low values of $\theta_{E}$ for $\mathrm{Gd}-\mathrm{O}$ subshells compared to that for the $\mathrm{Cr}-\mathrm{O}$ shell suggest that the $\mathrm{Gd}-\mathrm{O}$ bonds are weaker than $\mathrm{Cr}-\mathrm{O}$ bonds; i.e., $\mathrm{GdO}_{12}$ polyhedra are not as rigid as $\mathrm{CrO}_{6}$ octahedra. Furthermore, there is clear deviation of $\sigma_{0}^{2}$ from the expected behavior in the region near the magnetic/ferroelectric transition temperature, suggesting the presence of structural anomalies around the transition. The temperature-dependent variation of $\sigma^{2}$ for Gd$\mathrm{Cr}$ bond correlations along with fitting using Eq. (1) are shown in Fig. 4(b), giving $\sigma_{0}^{2}=0.0044(1)$ and $\theta_{E}=293(7) \mathrm{K}$ for $\mathrm{Gd}-\mathrm{Cr}_{1} / \mathrm{Cr}_{3}$ bonds and $\sigma_{0}^{2}=0.0015(3)$ and $\theta_{E}=428(6) \mathrm{K}$ for the $\mathrm{Gd}-\mathrm{Cr}_{2}$ bond. The $\sigma^{2}$ for $\mathrm{Gd}-\mathrm{Cr}_{1} / \mathrm{Cr}_{3}$ bonds which are in the $b c$ plane show an anomaly around the transition, possibly due to the Gd displacements caused by the magnetostriction effect associated with the $\mathrm{Gd}^{3+}-\mathrm{Cr}^{3+}$ interaction $[11,23]$, whereas the $\mathrm{Gd}-\mathrm{Cr}_{2}$ bond shows no anomalous behavior.

For a further understanding of the structural properties, we have extracted various bond lengths from EXAFS fitting and Rietveld refinement of the XRD pattern, and the temperature dependence of average $\mathrm{Cr}-\mathrm{O}$ bond lengths is depicted in Fig. 5(a). It is observed that the average bond lengths obtained from both EXAFS and XRD analysis are very close and show no anomalies around the transition temperature. Figures 5(b)$5(\mathrm{~d})$ represent the individual bond lengths $\left(\mathrm{Cr}-\mathrm{O}_{1} / \mathrm{O}_{2} / \mathrm{O}_{3}\right)$ extracted from the XRD analysis, in which $\mathrm{O}_{1}$ occupies the apex and $\mathrm{O}_{2} / \mathrm{O}_{3}$ occupy the base of the $\mathrm{CrO}_{6}$ octahedra. The bond length of $\mathrm{Cr}-\mathrm{O}_{1}$ contracts with decreasing temperature, while in the $a b$ plane the $\mathrm{Cr}-\mathrm{O}_{2}$ bond length decreases and the $\mathrm{Cr}-\mathrm{O}_{3}$ bond length increases with decreasing temperature. The $\mathrm{Cr}-\mathrm{O}_{3}$ bond shows anomalous behavior around the transition, indicating the magnetostriction effect, consistent with the Raman spectroscopy study as reported earlier [11]. The temperature-dependent variation of the bond angles with temperature is shown in Figs. 5(e) $-5(\mathrm{~g})$. With decreasing temperature the axial angle $\left(\mathrm{Cr}-\mathrm{O}_{1}-\mathrm{Cr}\right)$ increases and one of the equatorial angles $\left(\mathrm{Cr}-\mathrm{O}_{2}-\mathrm{Cr}\right)$ decreases, whereas the other equatorial angle $\left(\mathrm{Cr}-\mathrm{O}_{3}-\mathrm{Cr}\right)$ increases. The bond angles also exhibit anomalous behavior near the transition temperature, suggesting that there exist distortions in the octahedra probably associated with the off-center displacements of oxygens via octahedral rotations [6,7]. It is known that the off-center displacement of oxygens, generally in the $a b$ plane, is the most important factor for the Dzyaloshinskii-Moriya interaction in the system $[24,25]$.

The temperature-dependent variation of $\mathrm{Gd}-\mathrm{O}$ and $\mathrm{Gd}-\mathrm{Cr}$ bond lengths obtained from EXAFS and XRD analyses are shown in Figs. 6(a) and 6(b), respectively. Except for $\mathrm{Gd}_{-} \mathrm{O}_{3}$, all the Gd-O and Gd-Cr bonds obtained from EXAFS analysis match reasonably well with that extracted from the Rietveld refinement of XRD. The Gd-O3 bond obtained from EXAFS fitting is shorter by $\sim 0.07 \AA$ than that determined from XRD. Discrepancies between EXAFS and diffraction results may 


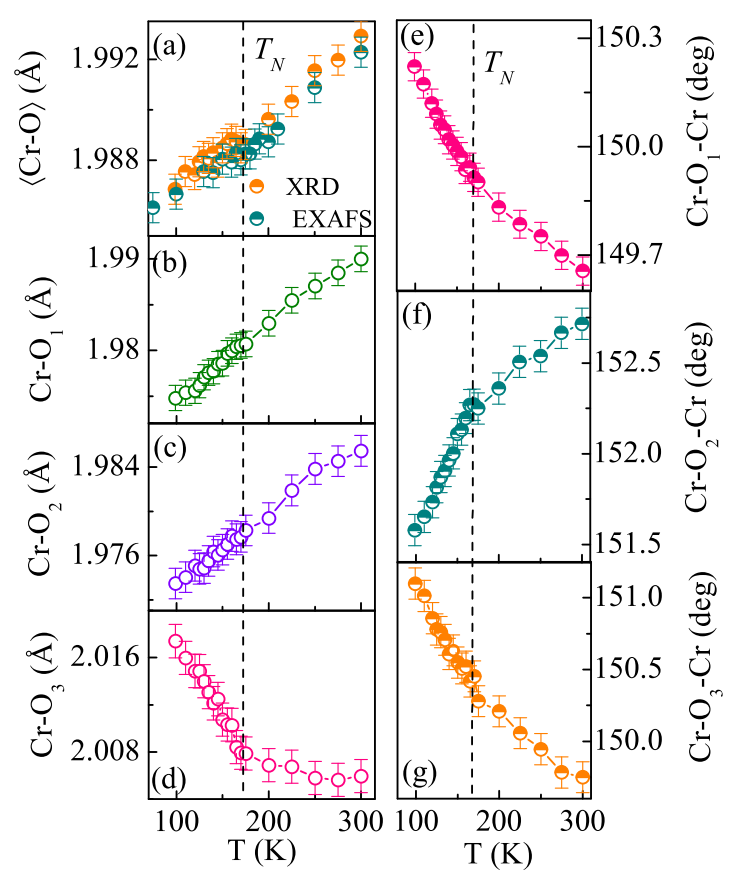

FIG. 5. (a) Temperature dependence of average $\mathrm{Cr}-\mathrm{O}$ atomic bond length in $\mathrm{GdCrO}_{3}$ obtained from EXAFS and XRD analyses. Temperature dependence of bond lengths $\left(\mathrm{Cr}-\mathrm{O}_{1} / \mathrm{O}_{2} / \mathrm{O}_{3}\right)$ and bond angles $\left(\mathrm{Cr}-\mathrm{O}_{1} / \mathrm{O}_{2} / \mathrm{O}_{3}-\mathrm{Cr}\right)$, obtained from Rietveld refinement of $\mathrm{XRD}$ are shown in (b)- $(\mathrm{g})$, respectively. The vertical dashed lines correspond to the magnetic/ferroelectric transition temperature.

arise from either physical or fictitious effects. The systematic errors in the EXAFS data analysis may originate from the correlation between distances and energy scale parameters. On the other hand, discrepancies can also be possible due to the differences between local and long-range structures as observed in various systems like $\mathrm{La}_{1-x} \mathrm{Sr}_{x} \mathrm{MnO}_{3}$ [26],

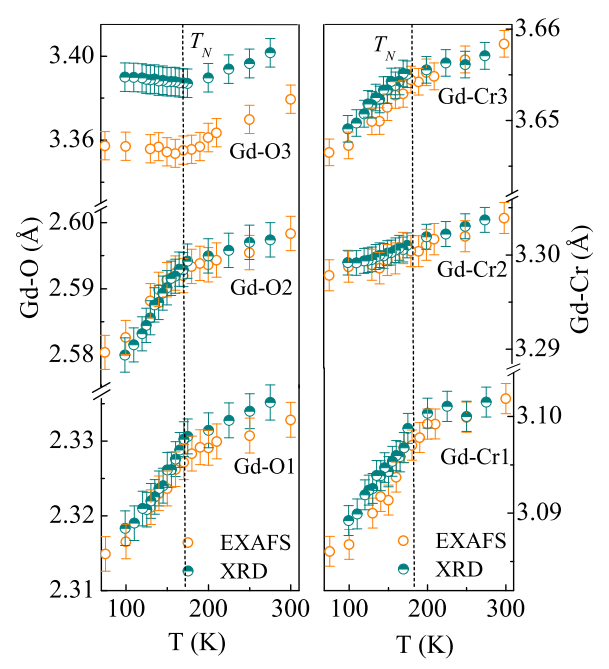

FIG. 6. Temperature dependence of (a) various Gd-O bond lengths and (b) Gd-Cr bond lengths obtained from EXAFS and XRD analyses. The vertical dashed lines correspond to the magnetic/ferroelectric transition temperature.
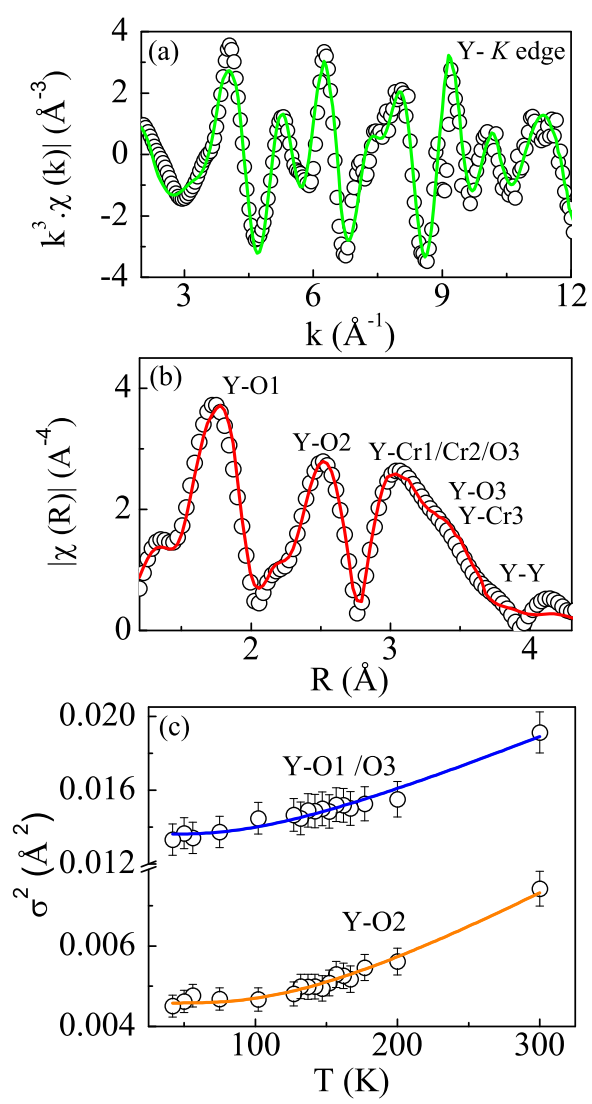

FIG. 7. (a) $k^{3}$-weighted EXAFS data in $k$ space and (b) magnitude of Fourier transform of the data at $\mathrm{Y} K$ edge acquired at room temperature for $\mathrm{YCrO}_{3}$, along with corresponding fittings (solid lines). Various contributions in different regions are marked in the figure. (c) Temperature dependence of MSRD $\left(\sigma^{2}\right)$ of Y-O bond correlations. The solid lines represent the fitting using the Einstein model [Eq. (1)].

$\mathrm{La}_{1-x} \mathrm{Ca}_{x} \mathrm{MnO}_{3}$ [27], $\mathrm{La} / \mathrm{PrCoO}_{3}$ [28], and $\mathrm{Na}_{0.5} \mathrm{Bi}_{0.5} \mathrm{TiO}_{3}$ [29]. In the present system, the nearly equal lengths of Gd-O3 and $\mathrm{Gd}-\mathrm{Cr}$ bonds may lead to the suppression of the $\mathrm{Gd}-\mathrm{O} 3$ contribution. However, the contraction is observed only for one distance (Gd-O3 bond), while all the other bond lengths show a general agreement between XRD and EXAFS results. Further, though there is a difference in the magnitude of the Gd-O3 bond length obtained from XRD and EXAFS analyses, their temperature evolutions are similar. This points towards the true shorter distance of the Gd-O3 bond length, which may be possibly due to the deviations of local structure from the average structure. The change in local structure is mainly associated with the Gd-O coordination sphere, whereas the $\mathrm{Cr}-\mathrm{O}$ coordination remains less affected, suggesting that the structural distortion is dominated by $\mathrm{Gd}$ displacements. Further, all the Gd-O bonds and $\mathrm{Gd}-\mathrm{Cr}$ bonds except $\mathrm{Gd}_{-} \mathrm{Cr}_{2}$ show slope changes around the transition temperature. This anomalous behavior can be understood through magnetostriction effect, which plays a role in ferroelectric distortion [11,23].

For comparison the EXAFS measurements were carried out on a similar chromite system, $\mathrm{YCrO}_{3}$. Figures 7 (a) and 7(b) depict $k^{3}$-weighted EXAFS data in $k$ space and magnitude of Fourier transform of the data, respectively, acquired at the Y $K$ 
edge at room temperature for $\mathrm{YCrO}_{3}$, along with corresponding fitting superimposed on these. The scattering contributions for atomic shells were derived and fitted considering the Pbnm crystal structure and $R$-factor was found to be $\sim 0.01$ for the entire (measured) temperature range. Fits were confined to the $k$ range of $3<k<12.5 \AA^{-1}$ and an $R$ range of $1.15<R<$ $4 \AA$ A. Here the EXAFS was fitted with three nearest-neighbor $\mathrm{O}$ shells of four coordinations each, three $\mathrm{Y}-\mathrm{Cr}$ shells with two, four, and two atoms, respectively, and a single Y shell consisting of four neighbors. A total of five $\sigma^{2}$ parameters were chosen in a similar way to that used for fitting Gd EXAFS in $\mathrm{GdCrO}_{3}$. The fitting of the temperature dependence of $\sigma^{2}$ for Y-O subshells using Eq. (1) is shown in Fig. 7(c). From the fitting, it is found that $\sigma_{0}^{2}=0.0083(2)$ and $\theta_{E}=341(7) \mathrm{K}$ for $\mathrm{Y}-\mathrm{O} 1 / \mathrm{O} 3$ bond distributions and $\sigma_{0}^{2}=0.0015(8)$ and $\theta_{E}=432(5) \mathrm{K}$ for the $\mathrm{Y}-\mathrm{O} 2$ bond distribution. The relatively low values of $\theta_{E}$ for $\mathrm{Y}-\mathrm{O}$ subshells indicate $\mathrm{YO}_{12}$ polyhedra are also not rigid enough, like $\mathrm{GdO}_{12}$ in $\mathrm{GdCrO}_{3}$. These results suggest a close analogy between $\mathrm{GdCrO}_{3}$ and $\mathrm{YCrO}_{3}$. However, the Gd-O environment (three subshells with four, two, and six oxygens, respectively) in $\mathrm{GdCrO}_{3}$ is different from the Y-O environment (three subshells with four oxygens each) in $\mathrm{YCrO}_{3}$, which leads to an orthorhombic $P n a 2_{1}$ structure in $\mathrm{GdCrO}_{3}$ in contrast to the monoclinic $P 2_{1}$ structure in $\mathrm{YCrO}_{3}[10,11]$. The good fitting of EXAFS data using the $\mathrm{Pbnm}$ structure is possibly due to the tiny distortions in these systems, giving rise to very weak polarizations $\left(\mathrm{GdCrO}_{3}, \sim 0.7\right.$ $\left.\mu \mathrm{C} / \mathrm{cm}^{2} ; \mathrm{YCrO}_{3}, \sim 3 \mu \mathrm{C} / \mathrm{cm}^{2}\right)[5,10]$.

The distortion in the structure is associated with the offcentering displacement of $\mathrm{Gd}$ atoms together with octahedral rotations via movements of the oxygen ions, which lift certain symmetries of the centrosymmetric Pbnm structure [6,7] and possibly stabilize a local noncentrosymmetric Pna $2_{1}$ structure. Figure 8 illustrates a schematic representation of the displacement of oxygens around the $\mathrm{CrO}_{6}$ octahedron (octahedral rotation), as indicated by the arrows, and dashed circles represent the possible displacement of $\mathrm{Gd}$ atoms. The distortion is dominated mainly by Gd displacements, indicating that $\mathrm{Gd}-\mathrm{O}$ bond polarization plays a major role in driving ferroelectricity. Moreover, it is found that $\mathrm{GdCrO}_{3}$ prefers $P n a 2_{1}$ symmetry as discussed earlier, suggesting that some sort of long-range positional disorder is always present in the system [11]. The magnetic coupling leads to extra distortion in the system via the magnetostriction effect and

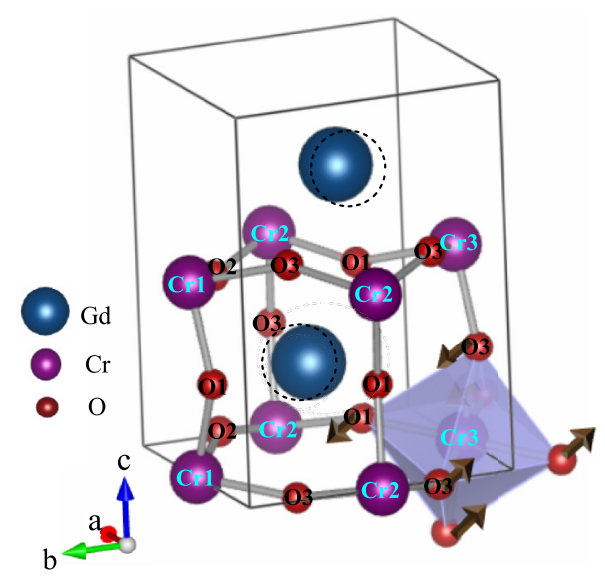

FIG. 8. Visualization of displacement of oxygens around the $\mathrm{CrO}_{6}$ octahedron (octahedral rotation), as indicated by the arrows and dashed circles, representing possible displacement of $\mathrm{Gd}$ atoms in $\mathrm{GdCrO}_{3}$.

plays a complementary role in the enhancement of ferroelectric polarization $[8,11,23]$.

\section{CONCLUSION}

In conclusion, through detailed structural investigations using temperature-dependent XRD and EXAFS studies, it is found that $\mathrm{CrO}_{6}$ octahedra in $\mathrm{GdCrO}_{3}$ are rather rigid and the $\mathrm{Gd}-\mathrm{O}$ bonds are weaker than the $\mathrm{Cr}-\mathrm{O}$ bonds. Octahedral rotations along with $\mathrm{Gd}$ displacements lead to noncentrosymmetric $P n a 2_{1}$ structure in $\mathrm{GdCrO}_{3}$. The distortion is dominated by $\mathrm{Gd}$ displacements, indicating that $\mathrm{Gd}-\mathrm{O}$ bond polarization plays a major role in driving ferroelectricity in this system.

\section{ACKNOWLEDGMENTS}

S.M. would like to acknowledge Prof. S. D. Mahanti, Michigan State University, for his useful discussions. S.M., U.M., P.N., and D.T. would like to gratefully acknowledge financial support by the Department of Science and Technology (Government of India) provided with in the framework of the India@DESY collaboration. U.M. and D.T. would also like to thank International Centre for Theoretical Physics, Italy, under the ICTP-Elettra users program for the financial support for experiments at Elettra, Italy.
[1] N. Ortega, A. Kumar, J. Scott, and R. S. Katiyar, J. Phys.: Condens. Matter 27, 504002 (2015).

[2] M. M. Vopson, Crit. Rev. Solid State Mater. Sci. 40, 223 (2015).

[3] R. Ramesh and N. A. Spaldin, Nat. Mater. 6, 21 (2007).

[4] H. Palneedi, V. Annapureddy, S. Priya, and J. Ryu, Actuators 5, 9 (2016).

[5] B. Rajeswaran, D. I. Khomskii, A. K. Zvezdin, C. N. R. Rao, and A. Sundaresan, Phys. Rev. B 86, 214409 (2012).

[6] A. Ghosh, K. Dey, M. Chakraborty, S. Majumdar, and S. Giri, Europhys. Lett. 107, 47012 (2014).

[7] A. Ghosh, A. Pal, K. Dey, S. Majumdar, and S. Giri, J. Mater. Chem. C 3, 4162 (2015).
[8] A. Indra, K. Dey, A. Midya, P. Mandal, O. Gutowski, U. Rütt, S. Majumdar, and S. Giri, J. Phys.: Condens. Matter 28, 279601 (2016).

[9] H. J. Zhao, L. Bellaiche, X. M. Chen, and J. Íñiguez, Nat. Commun. 8, 14025 (2017).

[10] C. R. Serrao, A. K. Kundu, S. B. Krupanidhi, U. V. Waghmare, and C. N. R. Rao, Phys. Rev. B 72, 220101 (2005).

[11] S. Mahana, B. Rakshit, R. Basu, S. Dhara, B. Joseph, U. Manju, S. D. Mahanti, and D. Topwal, Phys. Rev. B 96, 104106 (2017).

[12] K. Ramesha, A. Llobet, T. Proffen, C. Serrao, and C. Rao, J. Phys.: Condens. Matter 19, 102202 (2007). 
[13] N. Ray and U. V. Waghmare, Phys. Rev. B 77, 134112 (2008).

[14] S. Mahana, U. Manju, and D. Topwal, AIP Conf. Proc. 1832, 130046 (2017).

[15] S. Mahana, U. Manju, and D. Topwal, J. Phys. D: Appl. Phys. 50, 035002 (2016).

[16] B. Ravel and M. Newville, J. Synchrotron Radiat. 12, 537 (2005).

[17] J. J. Rehr and R. C. Albers, Rev. Mod. Phys. 72, 621 (2000).

[18] J. J. Rehr, J. J. Kas, M. P. Prange, A. P. Sorini, Y. Takimoto, and F. Vila, C. R. Phys. 10, 548 (2009).

[19] A. L. Spek, Acta Crystallogr. Sect. D 65, 148 (2009).

[20] J. Ruiz-Fuertes, T. Bernert, D. Zimmer, N. Schrodt, M. KochMüller, B. Winkler, L. Bayarjargal, C. Popescu, S. MacLeod, and K. Glazyrin, Phys. Rev. B 96, 094101 (2017).

[21] J. Mustre de Leon, S. D. Conradson, I. Batistić, A. R. Bishop, I. D. Raistrick, M. C. Aronson, and F. H. Garzon, Phys. Rev. B 45, 2447 (1992).
[22] T. A. Tyson, M. Deleon, S. Yoong, and S.-W. Cheong, Phys. Rev. B 75, 174413 (2007).

[23] V. S. Bhadram, B. Rajeswaran, A. Sundaresan, and C. Narayana, Europhys. Lett. 101, 17008 (2013).

[24] F.-K. Chiang, M.-W. Chu, F. C. Chou, H. T. Jeng, H. S. Sheu, F. R. Chen, and C. H. Chen, Phys. Rev. B 83, 245105 (2011).

[25] T. Moriya, Phys. Rev. 120, 91 (1960).

[26] T. Shibata, B. A. Bunker, and J. F. Mitchell, Phys. Rev. B 68, 024103 (2003).

[27] C. Monesi, C. Meneghini, F. Bardelli, M. Benfatto, S. Mobilio, U. Manju, and D. D. Sarma, Phys. Rev. B 72, 174104 (2005).

[28] S. Pandey, S. Khalid, N. Lalla, and A. Pimpale, J. Phys.: Condens. Matter 18, 10617 (2006).

[29] B. N. Rao, L. Olivi, V. Sathe, and R. Ranjan, Phys. Rev. B 93, 024106 (2016). 